\title{
Abnormal proliferative response of the carotid artery of spontaneously hypertensive rats after angioplasty may be related to the depolarized state of its smooth muscle cells
}

S.L.D. Dalle Lucca ${ }^{1}$ J.J. Dalle Lucca ${ }^{1}$, A.C.R. Borges ${ }^{1}$,

S.S.M. Ihara ${ }^{2}$ and T.B. Paiva ${ }^{1}$
Departamentos de ${ }^{1}$ Biofísica and 2Patologia, Escola Paulista de Medicina, Universidade Federal de São Paulo, São Paulo, SP, Brasil

\section{Correspondence \\ T.B. Paiva \\ Departamento de Biofísica \\ EPM, UNIFESP \\ Rua Botucatu, 862 \\ 04023-062 São Paulo, SP \\ Brasil \\ Fax: + 55-11-571-5780 \\ E-mail: tbpaiva@biofis.epm.br \\ Research supported by FAPESP, CNPq and CAPES.}

Received June 16, 1999 Accepted May 3, 2000

\section{Abstract}

Hypertension is one of the major precursors of atherosclerotic vascular disease, and vascular smooth muscle abnormal cell replication is a key feature of plaque formation. The present study was conducted to examine the relationship between hypertension and smooth muscle cell proliferation after balloon injury and to correlate neointima formation with resting membrane potential of uninjured smooth muscle cells, since it has been suggested that altered vascular function in hypertension may be related to the resetting of the resting membrane potential in spontaneously hypertensive rats (SHR). Neointima formation was induced by balloon injury to the carotid arteries of SHR and renovascular hypertensive rats $(1 \mathrm{~K}-1 \mathrm{C})$, as well as in their normotensive controls, i.e., Wistar Kyoto (WKY) and normal Wistar (NWR) rats. After 14 days the animals were killed and the carotid arteries were submitted to histomorphometric and immunohistochemical analyses. Resting membrane potential measurements showed that uninjured carotid arteries from SHR smooth muscle cells were significantly depolarized $(-46.5 \pm 1.9 \mathrm{mV})$ compared to NWR $(-69 \pm 1.4 \mathrm{mV})$, NWR $1 \mathrm{~K}-1 \mathrm{C}(-60.8 \pm 1.6 \mathrm{mV})$, WKY $(-67.1 \pm 3.2 \mathrm{mV})$ and WKY $1 \mathrm{~K}-1 \mathrm{C}(-56.9 \pm 1.2 \mathrm{mV})$. The SHR arteries responded to balloon injury with an enhanced neointima formation (neo/media $=3.97 \pm$ 0.86 ) when compared to arteries of all the other groups (NWR $0.93 \pm$ 0.65 , NWR $1 \mathrm{~K}-1 \mathrm{C} 1.24 \pm 0.45$, WKY $1.22 \pm 0.32$, WKY $1 \mathrm{~K}-1 \mathrm{C} 1.15$ $\pm 0.74)$. Our results indicate that the increased fibroproliferative response observed in SHR is not related to the hypertensive state but could be associated with the resetting of the carotid smooth muscle cell resting membrane potential to a more depolarized state.
Key words

- SHR

- Renovascular hypertension

- Restenosis

- Smooth muscle cell

- Membrane potential 


\section{Introduction}

Blood vessel repair mechanisms designed to restore the vessel wall after damage caused by stress frequently escape self-limiting controls and result in lumen narrowing due to vascular smooth muscle cell (VSMC) proliferation. This is recognized as central to the pathology of both major forms of vascular disease, i.e., atherosclerosis and hypertension (1). In atherosclerosis, one of the most important cellular events is a fibroproliferative response consisting of the undifferentiation of VSMC into a synthetic form followed by migration to the lumen where they proliferate and secrete extracellular matrix proteins (2). The most widely used experimental model to study VSMC proliferation in vivo is balloon injury to the rat carotid artery which results in platelet aggregation and neointimal proliferation. The use of this model has shown that the proliferation involves several growth factors, such as platelet-derived growth factor, insulin growth factor, fibroblast growth factor, tumor growth factor, transforming growth factor, and angiotensin II (2-4). A common feature of growth factor-stimulated cells is an early change in membrane potential which leads in most cases to cell depolarization (5).

It is well established that an alteration in membrane potential plays a role in the activation of eukaryotic cell proliferation (5). Therefore, the physiological response of a given cell to a particular mitogen probably involves specific interactions between the mitogens and their receptors on the cell surface which lead to the opening of $\mathrm{Na}^{+}$channels causing depolarization and stimulation of the $\mathrm{Na}^{+} / \mathrm{H}^{+}$exchange mechanism promoting intracellular alkalinization. The depolarizing effect also causes $\mathrm{Ca}^{2+}$ influx through voltage-dependent $\mathrm{Ca}^{2+}$ channels which, combined with intracellular alkalinization, constitute an early mitogenic pathway signal (5).

The finding that spontaneously hyper- tensive rats (SRH) have enhanced neointima formation after balloon injury was attributed either to the hypertensive state itself (6) or to the higher concentration of angiotensin converting enzyme found in these animals (7). In SHR, structural and functional changes of the plasma membrane occur which may lead to increased sensitivity to various agonists, a deficient binding of calcium to plasma membrane, increased membrane permeability to sodium, potassium and calcium (8), and, consequently, to alterations in transmembrane ionic transport (9) and increased $\mathrm{Na}^{+} /$ $\mathrm{H}^{+}$exchange activity (10). Since membrane ionic permeability and active electrogenic transport are both important determinants for the maintenance of membrane potential in VSMC, it has been suggested that altered vascular function in hypertension could reset the membrane potential to a more depolarized level (11-13) which would favor cell proliferation in injured arteries from hypertensive animals.

To further understand the role of the hypertensive state in the increased proliferative response of SHR carotid arteries after injury, in the present study we examined the process of neointima formation in ballooninjured carotid arteries from two different animal models of hypertension, SHR and Goldblatt one kidney-one clip renovascular hypertensive rats $(1 \mathrm{~K}-1 \mathrm{C})$. The findings in the hypertensive animals were compared to their respective normotensive controls, Wistar Kyoto (WKY) and Wistar (NWR) rats. We also examined the possible relationship between carotid artery VSMC proliferation and the resting membrane potential of these cells before injury.

\section{Material and Methods}

\section{Animals}

Male SHR of the Okamoto-Aoki strain, and their normotensive controls (WKY and NWR from the Wistar Institute, Philadel- 
phia, PA, USA, inbred at Escola Paulista de Medicina, São Paulo, SP, Brazil), weighing $300 \pm 30 \mathrm{~g}$ were used.

\section{Blood pressure measurement}

Mean arterial blood pressure was measured before and two weeks after surgery using the tail-cuff method (Letica Instruments model LE5650/6, Barcelona, Spain). The cuff was inflated up to $250 \mathrm{mmHg}$ and the average of three consecutive measurements was determined.

\section{Renal hypertension}

Eight weeks before angioplasty, Goldblatt $1 \mathrm{~K}-1 \mathrm{C}$ renovascular hypertension was produced in WKY and NWR rats under ether anesthesia by applying a silver clip to the left renal artery with simultaneous nephrectomy of the right kidney as described before (14).

\section{Arterial injury model}

Animals were anesthetized by intraperitoneal injection of thionembutal $(20 \mathrm{mg} / \mathrm{kg}$; Abbott, São Paulo, SP, Brazil) and chloral hydrate $(30 \mathrm{mg} / \mathrm{kg}$; Merck, Darmstadt, Germany). The level of anesthesia was verified before and during surgery by evaluating vibrissa movement, tail tonus, and breathing rate in response to handling. If the animal did not attain an adequate anesthesia level in the first 7 min following administration, it was returned to the breeding colony for use in later experiments. This protocol ensured that animals remained under complete anesthesia for at least $40 \mathrm{~min}$.

The left common and external carotid arteries were exposed and, with the aid of a micrometer and magnifying glass, the external diameter of the common carotid artery was measured in situ and used for calibrating balloon expansion. A balloon catheter was passed through the external carotid artery and advanced into the aorta, inflated with saline, and pulled back to distend the common carotid artery to 1.2 times its external diameter. The latter procedure was repeated twice, after which the external carotid artery was ligated, and the incision sutured.

\section{Perfusion/fixation}

Fourteen days after balloon injury, the rats were anesthetized and transcardially perfused with phosphate-buffered saline (PBS) followed by $4 \%$ paraformaldehyde at a fixed perfusion pressure of $100 \mathrm{mmHg}$. The right and left common carotid arteries were removed, the adipose and connective tissue in the adventitia was excised, and the vessels were then divided into three equal segments and placed in $4 \%$ paraformaldehyde for further fixation. The segments were embedded in paraffin, cut with a rotary microtome $(5 \mu \mathrm{m})$, and stained with hematoxylin and eosin $(\mathrm{H} \& \mathrm{E})$ or picrosirius, or used for immunohistochemistry studies.

\section{Immunohistochemistry}

The artery slices were placed on glass slides previously treated with $2 \% 3$-aminopropyltriethoxysilane (Sigma Chemical Co., St. Louis, MO, USA), digested with $0.1 \%$ trypsin (Sigma) in PBS at $37^{\circ} \mathrm{C}$ for $12 \mathrm{~min}$, and blocked with normal bovine serum albumin (Sigma). The sections were then covered with anti-smooth muscle $\alpha$-actin as primary antibody (Sigma) for $18 \mathrm{~h}$ at $4^{\circ} \mathrm{C}$, washed with PBS, incubated with biotinylated secondary antibody and with streptavidin peroxidase conjugate (Dako LSAB kit, Dako Corporation, Carpinteria, CA, USA), and developed with $0.02 \% \mathrm{H}_{2} \mathrm{O}_{2}$ and $0.1 \%$ diaminobenzidine tetrachloride (Sigma). The slides were lightly counterstained with hematoxylin.

\section{Morphology/morphometry}

Cross-sectional rings stained with H\&E 
were used to determine the extent of neointima formation. Analysis was performed using a Zeiss Axiovert microscope (Zeiss, Jena, Germany) attached to an imaging system analyzer Image-1/Metaphorph (Universal Imaging Corp., Westerchester, PA, USA) at $100 \mathrm{X}$ magnification. Measurements were made by two independent operators using a double-blind protocol. The average of these measurements was used and they did not differ by more than $0.1 \%$.

\section{Cell density}

Cross-sectional rings that were stained with $H \& E$ were used to determine cell density. Analysis was performed using a KS300 imaging system (Kontron Elektronik GmbH, Image Analysis Division, Eching, Munich, Germany) in a manner similar to that described above. The areas of three different regions within the neointima from the same arterial segment were measured and the nuclei within each region counted. Cell density was determined by dividing the number of nuclei by the area measured (nuclei $/ \mu \mathrm{m}^{2}$ ) and is expressed as the average of three measurements for each segment.

\section{Membrane potential}

Microelectrodes were constructed as previously described (13) by pulling capillary tubes on a horizontal puller (Narishige model PN3, Tokyo, Japan). The pipettes were filled with $2 \mathrm{M} \mathrm{KCl}$ and had a tip resistance of 20-40 M $\Omega$. The electrodes were mounted on $\mathrm{Ag} / \mathrm{AgCl}$ half-cells on a micromanipulator (Leitz, Wetzlar, Germany) and connected to an electrometer (Biodyne model AM-2, Santa Monica, CA, USA). The signals were recorded with a potentiometric chart recorder (ECB model RB102, São Paulo, SP, Brazil).

Uninjured carotid rings were placed in a 2-ml perfusion chamber and superfused at a rate of $3 \mathrm{ml} / \mathrm{min}$ with $\mathrm{Krebs}$ solution at $37^{\circ} \mathrm{C}$. The impalements were made directly in the smooth muscle cells from the adventitial side. Membrane potentials were measured as previously described (13). The successful implantation of an electrode was determined by a sharp drop in voltage upon cell entry, a stable potential $( \pm 3 \mathrm{mV})$ for a minimum of $60 \mathrm{~s}$ after impalement, a sharp return to baseline upon exit, and a minimal change $(<10 \%)$ in microelectrode resistance after impalement.

\section{Statistical analysis}

Each artery was divided into three equal segments (upper, middle, and lower) whose areas did not differ when compared by oneway analysis of variance (ANOVA). Therefore, an average of the three segments was used as a single measurement for each artery. Neointima formation is reported as the neointimal area/medial area ratio (neo/media).

ANOVA was used to compare lumen, media, neointima areas, the neo/media ratios, cell densities, as well as the resting membrane potentials. The Student $t$-test was used to perform all pairwise comparisons in the presence of an overall statistically significant group difference. The type I experimental error rate was maintained at 0.05 .

All experimental procedures and the general handling of animals were carried out in compliance with the Guide for the Care and Use of Laboratory Animals (National Institutes of Health, publication 85-23).

\section{Results}

Balloon injury was performed in carotid arteries of weight-matched SHR, WKY, NWR, as well as WKY and NWR with renovascular hypertension (WKY $1 \mathrm{~K}-1 \mathrm{C}$ and NWR 1K-1C, respectively). Blood pressure measurements were made before and 14 days after balloon injury, and showed that the three hypertensive groups had significantly higher mean arterial blood pressure than 
their respective controls (Table 1).

Two weeks after balloon injury, histomorphometric analysis of cross-sectional segments demonstrated that medial hypertrophy occurred in right side uninjured arteries of both SHR and $1 \mathrm{~K}-1 \mathrm{C}$ rats when compared to uninjured arteries from normotensive controls (data not shown).

Histomorphometric analysis revealed that neointima formation occurred in all experimental groups (Table 1, Figure 1), but was approximately 4-fold greater in SHR than in all other groups, in most cases leading to complete luminal obstruction (Figure 1F). Measurements of cellular density of the neointima showed no significant difference among groups (Table 1).

Immunohistochemistry was performed to identify the presence of smooth muscle cells. Single-labeled immunohistochemistry using an antibody against smooth muscle $\alpha$-actin consistently labeled VSMC in the medial layer of uninjured controls as well as the media and neointima of injured arteries of all experimental groups (Figure 2). Picrosirius staining showed collagen in the neointima of the injured arteries (data not shown). Despite the increased neointima formation observed in SHR, its cellular and extracellular composition qualitatively did not differ from the other experimental groups. However, the increased fibroproliferative response to in- jury in SHR carotid arteries could be related to an abnormally functioning VSMC membrane (8), which alters the resting membrane potential. In fact, resting membrane potential measurements of uninjured carotid arteries showed that the SHR VSMC was significantly less polarized when compared to normotensive and renovascular hypertensive carotid arteries, which had virtually identical resting membrane potentials. This is shown in Figure 3, which also illustrates an inverse relationship between resting membrane potential and neointima formation.

\section{Discussion}

Hypertension is an important risk factor promoting atherosclerosis. How hypertension influences the development of atherosclerosis remains unknown, although it is clear that smooth muscle cell differentiation and proliferation play a central role in this process (1). Balloon injury to the rat carotid artery is a useful model to study vascular smooth muscle cell proliferation in vivo and, with due consideration, may be a valuable instrument to obtain further information on the pathophysiology of cardiovascular diseases characterized by the activation and proliferation of VSMC, such as hypertension, atherosclerosis, and restenosis after angioplasty.

Table 1 - Mean arterial blood pressure (MAP), mean body weight (MBW), and morphometric analysis of injured arteries from SHR, NWR, WKY and renovascular hypertensive rats (NWR 1 K-1C and WKY 1 K-1C).

All values represent the mean $\pm S D ; N$, number of animals in each group; lumen, luminal area; media, medial area; neointima, neointimal area; $\mathrm{CDN}$, cellular density of the neointima. $* \mathrm{P}<0.05$ vs all other groups (Student t-test).

\begin{tabular}{lccccccc}
\hline & $\mathrm{N}$ & $\begin{array}{c}\text { MAP } \\
(\mathrm{mmHg})\end{array}$ & $\begin{array}{c}\text { MBW } \\
(\mathrm{g})\end{array}$ & $\begin{array}{c}\text { Lumen } \\
\left(\mathrm{mm}^{2}\right)\end{array}$ & $\begin{array}{c}\text { Media } \\
\left(\mathrm{mm}^{2}\right)\end{array}$ & $\begin{array}{c}\text { Neointima } \\
\left(\mathrm{mm}^{2}\right)\end{array}$ & $\begin{array}{c}\text { CDN } \\
\left(\mathrm{cells} / \mathrm{mm}^{2}\right)\end{array}$ \\
\hline NWR & 8 & $114 \pm 24$ & $315 \pm 29$ & $117.5 \pm 57.3$ & $73.4 \pm 6.5$ & $67.8 \pm 4.2$ & $123.9 \pm 31.1$ \\
NWR 1K-1C & 9 & $194 \pm 33^{*}$ & $325 \pm 30$ & $71.4 \pm 38.2$ & $63.5 \pm 8.8$ & $79.0 \pm 5.4$ & $152.2 \pm 17.8$ \\
WKY & 9 & $128 \pm 24$ & $295 \pm 29$ & $73.9 \pm 24.6$ & $80.4 \pm 10.4$ & $98.2 \pm 3.3$ & $120.7 \pm 14.5$ \\
WKY 1K-1C & 9 & $201 \pm 26^{*}$ & $275 \pm 17$ & $94.0 \pm 10.4$ & $98.9 \pm 17.8$ & $113.4 \pm 9.9$ & $148.3 \pm 12.1$ \\
SHR & 8 & $194 \pm 18^{*}$ & $287 \pm 18$ & $0.4 \pm 0.7^{*}$ & $67.0 \pm 27.5$ & $266.3 \pm 31.2^{*}$ & $168.8 \pm 41.6$
\end{tabular}


The increased neointima formation observed in injured SHR carotid arteries was first demonstrated by Clowes and Clowes (6) who attributed this increase to the hypertensive state. More recently, Jandeleit-Dahm et al. (7) reported similar results and proposed that the fibroproliferative response may be due to the higher concentration of angiotensin converting enzyme found in these animals. To further understand the role of the hypertensive state in the increased proliferative response of SHR carotid arteries after injury, we also measured neointima formation in rats with Goldblatt $1 \mathrm{~K}-1 \mathrm{C}$ renovascular hypertension. The finding that in- jured SHR, but not $1 \mathrm{~K}-1 \mathrm{C}$ renovascular hypertensive carotid arteries responded with an augmented neointima formation when compared to normotensive controls strongly suggests that the hypertensive state is not a determining factor for the fibroproliferative response of SHR arteries after injury. Furthermore, our findings that there are no differences in neointimal composition among any of the groups studied demonstrate that the increase in cellular proliferation observed is probably related to genetic alterations in SHR VSMC membrane.

It has been suggested that the altered vascular function in SHR may be related to
Figure 1 - Representative crosssectional segments of uninjured controls and injured rat carotid arteries 2 weeks after balloon injury. A, Uninjured WKY; $B$, injured WKY; $C$, uninjured WKY $1 \mathrm{~K}-1 \mathrm{C}$; D, injured WKY 1K-1C; $\mathrm{E}_{\text {, }}$ uninjured SHR; $F$, injured SHR. $\mathrm{M}=$ Media, $\mathrm{NI}=$ neointima, $\mathrm{AdV}$ $=$ adventitia. Staining: hematoxylin and eosin. Note: Neointima formation resulted in luminal obstruction in SHR. Bar: $400 \mu \mathrm{m}$.
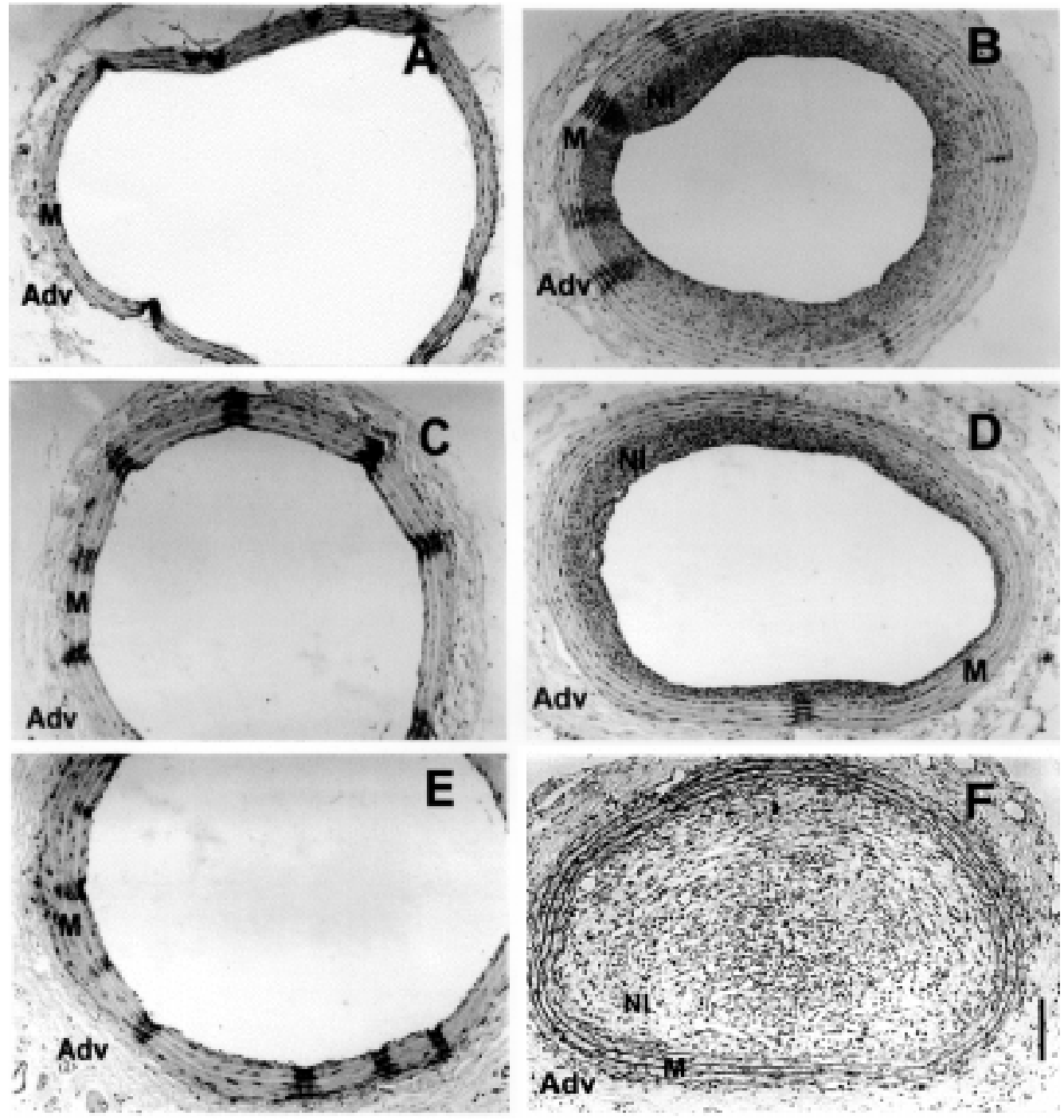
the resetting of the resting membrane potential (11-13,15). Several studies have shown that depolarization is among the first events in cells stimulated with most growth factors $(5,16)$. The subsequent increase in intracellular $\mathrm{Na}^{+}$concentration promotes a rapid activation of the $\mathrm{Na}^{+} / \mathrm{H}^{+}$exchanger and intracellular alkalinization, a condition that persists for up to $24 \mathrm{~h}$ (16). These processes are known to be associated with the initiation of the cellular proliferative cycle (5) and inhibitors of the $\mathrm{Na}^{+} / \mathrm{H}^{+}$exchange were shown to decrease neointima formation after rat carotid injury (17).

Studies relating VSMC membrane potential to hypertension have yielded contro-
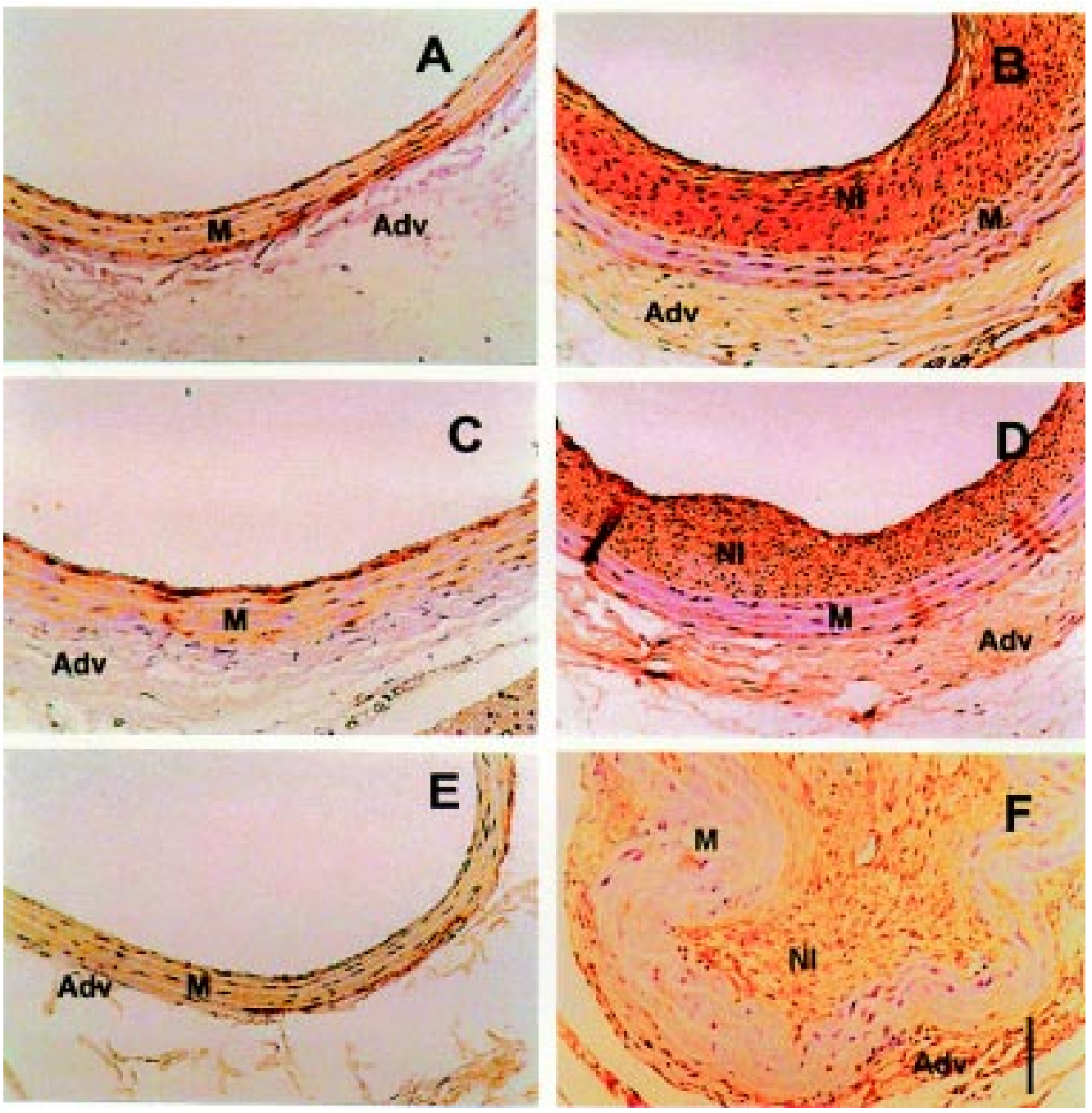

versial findings. Previous studies showed no membrane potential differences between WKY and SHR pulmonary arteries, portal veins, or rat tail arteries $(18,19)$. However, the methodology of these experiments was criticized by Cheung (11), who found that the development of hypertension in SHR was associated with a decrease in tail artery resting membrane potential when compared to WKY. Fujii et al. (12) and Feres et al. (13) demonstrated that, independently of the presence of a functional endothelium, SHR mesenteric artery smooth muscle cells are less polarized compared to WKY. The relation between VSMC proliferation and resting membrane potential was suggested by
Figure 2 - Representative immunohistochemistry of cross-sectional segments of uninjured controls and injured rat carotid arteries labeled with smooth muscle $\alpha$-actin, 2 weeks after balloon injury. $A$, Uninjured WKY; B, injured WKY; $C$, uninjured WKY 1K-1C; $D$, injured WKY 1K-1C; E, uninjured SHR; $F$, injured SHR. Background staining: hematoxylin. $\mathrm{M}=\mathrm{Me}-$ dia, $\mathrm{NI}=$ neointima, $\mathrm{Adv}=\mathrm{ad}-$ ventitia. Note: Neointima formation was heavily labeled with antibody against smooth muscle $\alpha$-actin in all groups. Bar: 200 $\mu \mathrm{m}$. 
Figure 3 - Bar graph showing neointima development two weeks after balloon injury (A) and resting membrane potential values (B) of uninjured carotid artery smooth muscle cells. Neo/media: Neointima formation; SHR: spontaneously hypertensive rats $(\mathrm{N}=8)$; NWR: normotensive Wistar-EPM rats $(\mathrm{N}=$ 8); WKY: Wistar Kyoto rats ( $\mathrm{N}=$ 9); NWR 1K-1C: renovascular hypertensive Wistar-EPM rats ( $\mathrm{N}$ = 9); WKY 1K-1C: renovascular hypertensive Wistar Kyoto rats $(\mathrm{N}=9) . * \mathrm{P}<0.05$ vs all other groups. Note: The increased neointima formation observed in injured SHR arteries could be associated with the depolarized values of resting membrane potential found in this rat strain.

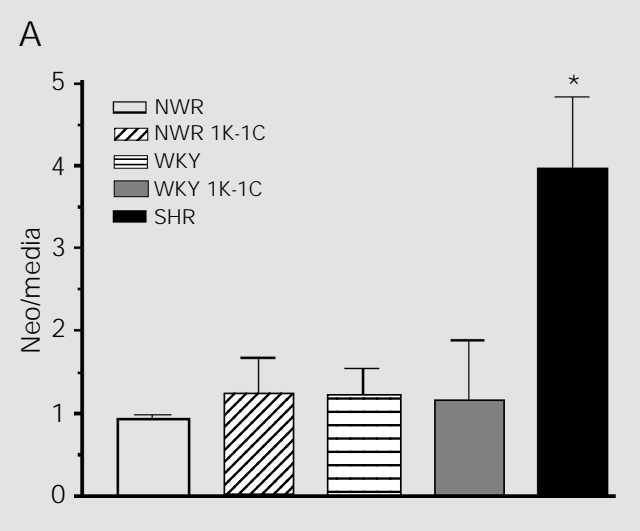

Blennerhassett et al. (20) based on data from SHR smooth muscle cell cultures. Low density cultures in the proliferative state showed depolarized membrane potentials, whereas confluent cultures of the same cells, whose mitotic process is interrupted, were hyperpolarized. Other studies in cultured SHR aortic VSMC reported an exaggerated response to several mitogenic stimuli (21-23). Snetkov et al. (24), using patch-clamp techniques, recently demonstrated that non-confluent VSMC suffer changes in $\mathrm{K}^{+}$channel conductance leading to depolarization and consequently to proliferation. In vivo, an indirect demonstration of the relationship between VSMC depolarization and proliferation is in the findings that the development of hypertension in SHR is paralleled by a decrease in membrane potential, and that this depolarization is reversed by antihypertensive treatments (11), which also prevent
B

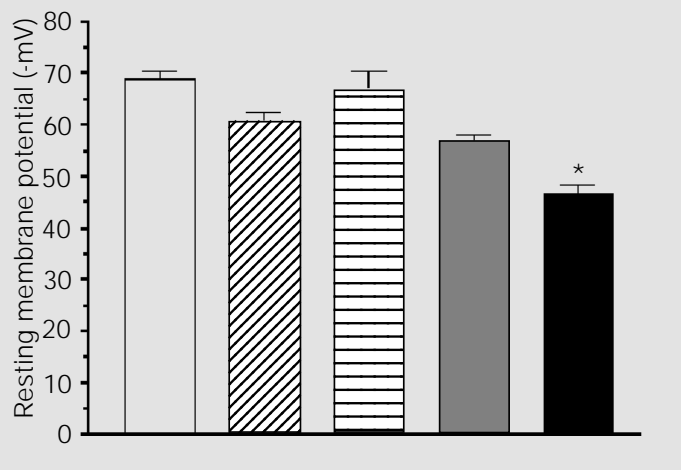

neointima formation after vascular injury in rats (7).

The present results, while confirming the less polarized state of the SHR cell membrane, do not support an association between membrane potential and hypertension since renovascular hypertensive animals, presenting blood pressure levels similar to those of SHR, were found to have normal carotid VSMC membrane potentials. Furthermore, the fact that renovascular hypertensive animals, in contrast to SHR, did not differ from normotensive controls in their response to balloon injury strongly suggests that genetic differences in SHR are probably an important determining factor of neointimal growth following arterial injury. Apparently, resetting of the resting membrane potential to a more depolarized state in SHR VSMC may account for this increased fibroproliferative response.

\section{References}

1. Schwartz SM, Campbell GR \& Campbell J H (1986). Replication of smooth muscle cells in vascular disease. Circulation Research, 58: 427-444.

2. Ip J H, Fuster V, Badimon L, Badimon J, Taubman MB \& Chesebro J H (1990). Syndromes of accelerated atherosclerosis: role of vascular injury and smooth muscle cell proliferation. J ournal of the American College of Cardiology, 15: 1667-1687.

3. Ross R (1993). The pathogenesis of atherosclerosis: a perspective for the 1990's.
Nature, 362: 801-809.

4. Schwartz SM, Heimark RL \& Majesky MW (1990). Developmental mechanisms underlying pathology of arteries. Physiological Reviews, 70: 1177-1209.

5. Rothenberg P, Reuss L \& Glaser L (1982). Serum and epidermal growth factor transiently depolarize quiescent BSC-1 epithelial cells. Proceedings of the National Academy of Sciences, USA, 79: 77837787.

6. Clowes AW \& Clowes MM (1980). Influ- ence of chronic hypertension on injured and uninjured arteries in spontaneously hypertensive rats. Laboratory Investigation, 43: 535-541.

7. J andeleit-Dahm K, Burrell LM, J ohnston Cl \& Koch KM (1997). Elevated vascular angiotensin converting enzyme mediates increased neointima formation after balloon injury in spontaneously hypertensive rats. J ournal of Hypertension, 15: 643650.

8. Bohr DF \& Webb RC (1988). Vascular 
smooth muscle membrane in hypertension. Annual Review of Pharmacology and Toxicology, 28: 389-409.

9. J ones AW (1973). Altered ion transport in vascular smooth muscle from spontaneously hypertensive rats. Influences of aldosterone, norepinephrine, and angiotensin. Circulation Research, 33: 563-572.

10. Foster CD, Honeyman TW \& Scheid CR (1992). Alterations in $\mathrm{Na}^{+}-\mathrm{H}^{+}$exchange in mesenteric arteries from spontaneously hypertensive rats. American J ournal of Physiology, 262: H1657-H1662.

11. Cheung DW (1984). Membrane potential of vascular smooth muscle and hypertension in spontaneously hypertensive rats. Canadian J ournal of Physiology and Pharmacology, 62: 957-960.

12. Fujii $K$, Tominaga $M$, Ohmori S, Kobayashi K, Koga T, Takata Y \& Fujishima M (1992). Decreased endothelium-dependent hyperpolarization to acetylcholine in smooth muscle of the mesenteric artery of spontaneously hypertensive rats. Circulation Research, 70: 660-669.

13. Feres T, Borges ACR, Silva EG, Paiva ACM \& Paiva TB (1998). Impaired function of alpha-2 adrenoceptors in smooth muscle of mesenteric arteries from spontaneously hypertensive rats. British J ournal of Pharmacology, 125: 1144-1149.

14. Schaffenburg CA (1959). Device to control contraction of main renal artery for production of hypertension in small animals. Proceedings of the Society for Experimental Biology and Medicine, 101: 676-677.

15. Silva EG, Frediani-Neto E, Ferreira AT, Paiva ACM \& Paiva TB (1994). Role of $\mathrm{Ca}^{2+}$-dependent $\mathrm{K}$ channels in the membrane potential and contractility of aorta from spontaneously hypertensive rats. British J ournal of Pharmacology, 113: 1022-1028.

16. Berk BC, Elder E \& Mitsuka M (1990). Hypertrophy and hyperplasia cause differing effects on vascular smooth muscle cell $\mathrm{Na}^{+} / \mathrm{H}^{+}$exchange and intracellular $\mathrm{pH}$. J ournal of Biological Chemistry, 265: 19632-19637.

17. Mitsuka M, Nagae M \& Berk BC (1993). $\mathrm{Na}^{+} / \mathrm{H}^{+}$exchange inhibitors decrease neointimal formation after rat carotid injury. Effects on smooth muscle cell migration and proliferation. Circulation Research, 73: 269-275.

18. Hermsmeyer K (1976). Electrogenesis of increased norepinephrine sensitivity of arterial vascular muscle in hypertension. Circulation Research, 38: 362-367.

19. Kuriyama H \& Suzuki H (1978). Electrical property and chemical sensitivity of vascular smooth muscles in normotensive and spontaneously hypersensitive rats. J ournal of Physiology, 285: 409-424.

20. Blennerhassett MG, Kannan MS \& Gar- field RE (1989). Density-dependent hyperpolarization in cultured aortic smooth muscle cells. American J ournal of Physiology, 256: C644-C651.

21. Hamada M, Nishio I, Baba A, Fukuda K, Takeda J, Ura M, Hano T, Kuchii M \& Masuyama $Y$ (1990). Enhanced DNA synthesis of cultured vascular smooth muscle cells from spontaneously hypertensive rats. Difference of response to growth factor, intracellular free calcium concentration and DNA synthesizing cell cycle. Atherosclerosis, 81: 191-198.

22. Agrotis A, Bray PJ, Saltis J \& Bobik A (1993). Vascular smooth muscle cell proliferation in SHR and WKY rats: evidence for specific differences in growth inhibitory regulatory mechanisms. Clinical and Experimental Pharmacology and Physiology, 20: 327-330.

23. Agrotis A, Saltis J \& Bobik A (1994). Effect of transforming growth factor-beta 1 on platelet-derived growth factor receptor binding and gene expression in vascular smooth muscle cells from SHR and WKY rats. Clinical and Experimental Pharmacology and Physiology, 21: 145-148.

24. Snetkov VA, Hirst SJ \& Ward J P (1996). Ion channels in freshly isolated and cultured human bronchial smooth muscle cells. Experimental Physiology, 81: 791804. 